\title{
SPECIAL \\ The Young Psychiatrists' Network: between past and future
}

\author{
D. Krupchanka ${ }^{1}$ and T. $\operatorname{Los}^{2}$
}

'Senior Researcher, Department of Social Psychiatry, National Institute of Mental Health, Tectich Chepublic: Affiliated Researcher, Centre for Global Mental Health, Institute of Psychiatry, Psychology and Neuroscience, King's College London, UK; email dzmitry. krupchanka@kclac.uk

${ }^{2}$ Researcher, Department of Population Health, London School of Hygiene and Tropical Medicine, London, UK
The Young Psychiatrists' Network is a movement of early-career psychiatrists that was established in $\mathbf{2 0 0 9}$ with the principles of peer regulation, lack of hierarchy and transparency. From humble beginnings as a small group of professionals in Eastern Europe, the Network is expanding into a globally inclusive platform. Future challenges, such as retention of the Network's core values, are now being debated.

In recent years, there has been increasing recognition of the urgent need to address the global workforce crisis in mental healthcare (Patel, 2009; Collins et al, 2011; Kakuma et al, 2011). Global collaborative efforts are needed to overcome this challenge through task-sharing, capacity-building and promotion of leadership (Saxena et al, 2007; Kakuma et al, 2011).

Strengthening professional networks and empowering young specialists are essential ingredients to ensure professional development and ongoing peer and academic support for psychiatrists. There are several organisations addressing these needs around the globe. However, there is a growing demand to tighten links and promote collaboration between them to broaden peer communication in the mental health sector. In light of this, an international platform for professional development has been established, called the Young Psychiatrists' Network (YPN) (Bendix et $a l$, 2011a). The Network represents an independent movement that aims to provide support to psychiatrists early in their careers and to promote leadership in mental health.

Since its conception in 2009, the YPN has been an entirely peer-regulated initiative led by young psychiatrists. Until now, the Network has not had a rigid structure and rules prescribing its functioning, development and collaborations. Instead, a flexible self-assembled group of active members makes decisions through negotiation and mutual respect. This absence of hierarchy and bureaucracy promotes equality among members and openness to ideas; the institutional culture is welcoming of innovation in psychiatry and is inclusive of all interested. The YPN has predominantly gathered members who are psychiatry trainees and earlystage practitioners, aged between 20 and 40 years. The absence of strict eligibility criteria has allowed a wide range of professionals to collect and connect during annual meetings, thereby strengthening professional relationships and enabling learning from diverse perspectives.
The guiding principles of the YPN are presented in Box 1. These principles make it possible for the YPN to sensitively address individual, professional and educational needs, while inspiring the involvement of all members. Crucially, the YPN welcomes the active engagement and involvement of people living with mental illness as experts, and aims to promote partnership in the design, development and delivery of psychiatric care. The YPN collaborates with a number of mental health organisations, national associations and serviceuser movements but maintains independence, without establishing formal ties.

The activities of the YPN consist of two main elements: continuous networking and annual meetings. Continuous networking occurs via online forums, personal meetings and activities that focus on the specific educational and professional needs of young psychiatrists, thereby facilitating knowledge exchange and the building of professional relationships, and enhancing research opportunities. The main purposes of the annual meetings are to learn from the contributions of the participants, to consolidate professional relationships and to empower young psychiatrists.

\section{The history of the Network}

The YPN was launched in 2009, when it was named Young Psychiatrists Eastern Europe, as an initiative of the Swedish Eastern Europe Committee to address the needs and concerns of young psychiatrists in the Baltic Sea Region and Belarus (Bendix et al, 2010) and to promote networking between young psychiatrists from Lithuania, Belarus, Russia and Sweden.

The first meeting focused on psychiatric diagnosis, and took place in 2010 in Vilnius, Lithuania (Bendix et al, 2011a). It was attended by 50 young psychiatrists from 10 European countries, who developed the innovative idea of a network outside of established organisations - a network created by young psychiatrists for young psychiatrists.

The second YPN meeting was held in Riga, Latvia, in 2011 (Bendix et al, 2011b). The meeting was supported by Riga Stradins University, the Swedish Psychiatric Association and the World Psychiatric Association, enabling 90 participants from 18 countries to attend. The agenda focused on personality disorders and coercive care in psychiatry. Young psychiatrists were invited to participate in workshops, present posters, give oral presentations and visit the Riga Centre of Psychiatry and Addiction Disorders. In anonymous feedback (response rate $75 \%)$ the vast majority $(96 \%)$ stated that the meeting had had a positive impact on them. 
Box 1. The vision, mission and values of the Young Psychiatrists' Network

Vision

Global development of psychiatry through close cooperation of young psychiatrists worldwide, expanding knowledge and sharing experiences, giving the means to influence psychiatric care at local and international levels.

\section{Mission}

Facilitating the evolution of young psychiatrists from different parts of the world, and improvement of their knowledge, skills and abilities through close interactions, international collaboration, sharing experiences and expertise.

Values

Striving to identify and communicate the highest level of clinical care, promoting the rights of people with mental illness, national diversity, cultural tradition, and working towards destigmatising psychiatric illness in society. The interaction between members of the YPN is grounded in mutual respect, openness, friendship and lack of a hierarchy, thereby encouraging an open dialogue and a friendly atmosphere. functioning was discussed during the meeting in Greece. All active members assembled to negotiate and introduce the first bylaws and policies, addressing YPN governance and future directions. The topics discussed included membership regulations, board elections, national coordinators' roles and responsibilities, complaint procedures and finances.

\section{The future of the Network}

The ongoing international expansion of the YPN has seen new members from various countries beyond Europe. These recent developments have placed the YPN at a crossroads, where the future of the movement must be carefully considered. Broadening the YPN demands reorganisation of its structure without interfering with its core values and vision. While it is vital that the YPN maintains its principles, it also recognises the importance of balancing these with the need to reach out to more young psychiatrists who can benefit from and contribute to the Network, in turn positively affecting psychiatric practice.

\section{Conclusions}

The YPN is a fast-growing movement that engages and inspires young psychiatrists to adopt direct roles in capacity-building, promoting leadership and networking within psychiatry. It has rapidly grown from a small group of psychiatrists in Eastern Europe into an international global initiative. However, this demands a restructuring of the Network's organisational framework. The question of how to restructure the YPN has yet to be answered. There is a need for the unique vision of the YPN to evolve in a way that allows it to maintain its valued principles, and the solution will be found through international collaboration.

For more information, please visit the YPN website (http://www.ypsnet.org).

\section{References}

Bendix, M., Olofsson, M. \& Hanson, J. (2010) Young psychiatrists meetings. Tidskriften för Svensk Psykiatri, 3, 80-81.

Bendix, M., Paravaya, O., Kochetkov, Y., et al (2011a) Young psychiatrists' meetings in Eastern Europe - networking for the development of psychiatric training, education and service. World Psychiatry, 10, 239.

Bendix, M., Jauhar, S., Smirnova, D., et al (2011b) 2nd Young Psychiatrists' Network meeting - Riga 2011: moving together towards new horizons. Available at http://www.wpanet.org/detail. php?section_id=22\&content_id=1046 (accessed December 2015).

Collins, P. Y., Patel, V., Joestl, S. S., et al (2011) Grand challenges in global mental health. Nature, 475, 27-30

Kakuma, R., Minas, H., van Ginneken, N., et al (2011) Human resources for mental health care: current situation and strategies for action. Lancet, 378, 1654-1663.

Patel, V. (2009) The future of psychiatry in low- and middle-income countries. Psychological Medicine, 39, 1759-1762.

Saxena, S., Thornicroft, G., Knapp, M., et al (2007) Resources for mental health: scarcity, inequity, and inefficiency. Lancet, 370 , 878-889.

Sinha, S. K., Bezborodovs, N., Krupchanka, D., et al (2013) Asian federation of early career psychiatrists: building bridges and expanding horizons. Asian Journal of Psychiatry, 6, 93-94.

Smirnova, D. \& Krupchanka, D. (2012) Early career psychiatrists corner: third Young Psychiatrists' Network meeting: stigma in YPs' perspective. Asian Journal of Psychiatry, 5, 208. 